\title{
Avaliando o Desempenho de uma Rede Aeronáutica Tolerante a Interrupções Programadas para Comunicação dos Passageiros Aéreos
}

\author{
Rafael de A. Silva ${ }^{1}$, Leandro de M. Sales ${ }^{1}$, Alessandro Anzaloni ${ }^{2}$ \\ ${ }^{1}$ Instituto de Computação - Universidade Federal de Alagoas (UFAL) \\ Caixa Postal 95 - 57.072-970 - Maceió - AL - Brasil \\ ${ }^{2}$ Departamento de Eletrônica - Instituto Tecnológico de Aeronáutica (ITA) \\ Caixa Postal 6022 - 12.228-900 - São José dos Campos - SP - Brasil \\ \{rafael, leandroal\}@ic.ufal.br, anzaloni@ita.br
}

\begin{abstract}
This paper describes and evaluates the SynFlight approach for aeronautical telecommunication networks. This approach enables a higher tolerance with disruptions as an aircraft perceives a specific air route. We utilize an discrete-event aeronautical simulator to perform a comparative simulation between this approach and classical protocols such as TCP and DTN. The following metrics are utilized in this work: (i) number of retransmissions; (ii) restart delay of transmission; (iii) level of energy consumption; and (iv) goodput. The results reveal the SynFlight approach presents a higher performance than classical protocols in scenarios with gaps over network coverage.
\end{abstract}

Resumo. Este artigo descreve e avalia a abordagem SynFlight para redes de telecomunicação aeronáutica. Esta abordagem permite uma maior tolerância com interrupções na transmissão quando uma aeronave percorrer uma determinada rota aérea. Utiliza-se um simulador aeronáutico para realizar uma simulação comparativa entre esta abordagem e protocolos clássicos como o TCP e o DTN. As seguintes métricas são utilizadas neste trabalho: (i) número de retransmissões; (ii) atrasos de reinício da comunicação; (iii) nível do consumo de energia; $e$ (iv) goodput. Os resultados revelam que a abordagem SynFlight apresenta um desempenho superior aos protocolos convencionais em cenários com falhas na cobertura de rede.

\section{Introdução}

Nos últimos anos, as Redes Tolerantes a Atrasos / Interrupções (Delay / Disruption Tolerant Networks ou DTN) têm sido um tema de crescente discussão e relevância na literatura. Segundo Fall (2003), estas redes são formadas por arquiteturas regionais ou segmentadas de redes conectadas através de gateways com armazenamento persistente, quebrando a ideia de transmissão fim-a-fim proposta por protocolos de transporte como o TCP. Uma DTN é uma arquitetura flexível, podendo utilizar a pilha de protocolos TCP/IP ou ser formada por protocolos específicos, sendo o protocolo principal denominado Bundle Protocol (BP) [Caini et. al. 2011]. Este protocolo permite que uma rede DTN armazene temporariamente as mensagens trafegadas em sua rede através de um mecanismo de armazenamento e repasse (conhecido como store-and-forward). A implementação desta rede pode ser definida em três camadas da arquitetura TCP/IP: (i) 
entre as camadas de enlace e transporte; (ii) na camada de aplicação (formando uma rede sobreposta (overlay); e (iii) na camada de transporte. A DTN tem sido aplicada em redes militares, interplanetárias, ad-hoc, móvel, veicular, civis terrestres e de sensores.

A arquitetura DTN propicia uma maior tolerância na transmissão de dados em cenários considerados desafiadores. Tal tolerância é provida pelo mecanismo de retransmissão do BP. Quando houver interrupções na comunicação, o mecanismo agirá da seguinte maneira [Caini et. al. 2009]: o mecanismo verifica o estado atual do socket do TCP toda vez que desejar transmitir dados. Se este socket não estiver ativa, o BP instancia um temporizador de 30 segundos e aguarda até que a mesma esteja ativa novamente. Caso o temporizador expire e o socket ainda não estiver disponível, o BP encerra a sessão do TCP. A partir deste ponto, o BP tenta iniciar uma nova sessão do TCP após um certo intervalo, enviando um segmento SYN e esperando a sua confirmação de entrega pelo receptor até que o número máximo de retransmissões (com parâmetros menores do que o TCP com sessão ativa) seja alcançado. Em caso de falha, o BP dobra o intervalo de tentativas e solicita novamente ao TCP a abertura de uma nova sessão até alcançar um patamar pré-definido (e.g. 24 horas). Depois disso, o protocolo BP passa a função de verificação da disponibilidade da rede para um algoritmo específico de descoberta da rede. Portanto o TCP é encerrado e um mecanismo próprio de retransmissão é acionado para reestabelecer uma nova sessão do TCP.

Embora efetiva no quesito tolerância, a arquitetura DTN gera um alto número de retransmissões desnecessárias em cenários com interrupções frequentes, consumindo energia do sistema e reduzindo a capacidade de transmissão do TCP. Além disso, o reinício da comunicação não é imediato - há um atraso referente ao modo de funcionamento do DTN, que só transmite em ciclos (i.e quando um temporizador expirar). Tais cenários são possíveis quando não há cobertura completa da rede ao longo de uma determinada região, o que pode ser devido a fatores como falhas em dispositivos de rádio, interferências entre as estações ou aeronaves, distribuição irregular das estações, restrições energéticas do sistema de comunicação ou pelo posicionamento inadequado das antenas de transmissão/recepção.

Um dos cenários mais críticos no quesito cobertura da rede é o de uma rede de telecomunicação aeronáutica (Aeronautical Telecommunication Network ou ATN) baseada em serviços de comunicação para passageiros aéreos (Air Passenger Communications ou APC). O padrão ATN [Schnell e Scalise 2007] é capaz de integrar diferentes tecnologias de comunicação aeronáutica tais como redes de satélites, plataformas de alta altitude, redes mesh entre aeronaves ou redes de estações aéreoterrestres (Air to ground ou ATG) [Schnell e Scalise 2007]. Dentre as tecnologias citadas, a utilização de estações ATG para fornecer serviços APC tem sido explorada por algumas empresas e fabricantes, ganhando destaque nos últimos anos. Em países com grandes extensões territoriais como o Brasil, a cobertura deste tipo de rede poderá ser prejudicada devido ao alto custo de implantação, além das dificuldades encontradas devido a terrenos irregulares, florestas ou regiões de difícil acesso. Manter os serviços dos usuários conectados com a porção terrestre da rede é um desafio para sistemas deste tipo, pois centenas de usuários utilizam aplicações simultaneamente, aumentando a complexidade de gerenciamento.

Este artigo investiga a abordagem SynFlight [Silva et al. 2014] para estabelecer redes conectadas com desempenho maior do que as redes tradicionais de comunicação aeronáutica. O SynFlight estabelece uma arquitetura que leva em conta informações 
relativas ao voo e as usam para estimar o tempo de cada transmissão ou interrupção ao longo de uma rota aérea. Portanto, o mesmo pode ser utilizado como abordagem para reduzir os efeitos das interrupções no cenário aeronáutico. Para avaliar o desempenho do TCP, da solução DTN e da proposta SynFlight, utiliza-se o simulador de redes aeronáuticas (Aeronautical Network Simulator ou ANS) [Ehammer e Rokitansky 2011] e a ferramenta MATLAB para tratar e analisar os dados obtidos por este simulador. A adoção do SynFlight melhora o desempenho da transmissão aeronáutica em relação ao protocolo TCP e a solução DTN em vários quesitos como capacidade de transmissão, reação a interrupções e agilidade na transferência de dados. Isto torna a eficiência do TCP próxima das condições ideais de transmissão (i.e. transmissão à taxa máxima durante todo o período de conectividade).

O restante deste trabalho é organizado da seguinte maneira. A Seção 2 apresenta a fundamentação teórica necessária para a compreensão deste trabalho. A Seção 3 descreve o sistema de comunicação proposto, com seus mecanismos, entidades e arquitetura. A Seção 4 descreve a análise de desempenho realizada neste trabalho, apresentando os parâmetros de desempenho utilizados, a metodologia de simulação e os resultados obtidos. A Seção 5 apresenta as considerações finais, apontando as contribuições e as propostas para padronização pela IETF.

\section{Fundamentação Teórica}

A arquitetura ATN apresenta um significativo avanço no controle operacional da aeronave e do tráfego aéreo, pois é possível, por meio desta, entregar serviços de voz e dados utilizando quaisquer enlaces que estejam disponíveis ao longo de um voo. A primeira versão desta arquitetura é baseada no modelo de referência OSI, sendo descrita nos documentos 9705/9880 do ICAO. A segunda versão desta arquitetura é baseada na suite de protocolos da Internet ou IPS, sendo descrita no documento 9896 do ICAO. A arquitetura ATN/IPS permite que os serviços de gerenciamento do tráfego aéreo global possam ser baseados em aplicações comerciais recentemente utilizadas na Internet, como aplicações VoIP, streaming de vídeos, programas de transferência de arquivos, entre outros.

\subsection{Requisitos de uma rede ATN/IPS}

O manual 9896 do ICAO define os seguintes critérios para o estabelecimento de uma rede ATN/IPS [ICAO 2011]: (i) que os nós da rede implementem os protocolos de transporte TCP e UDP; (ii) que os nós implementem extensões para garantir alta performance, conforme abordado no RFC 1323 [Jacobson, Braden e Borman 2014]; (iii) que os domínios administrativos da rede utilizem serviços diferenciados para prover QoS às aplicações e serviços desta rede, conforme especificado no RFC 2475 [Blake et al 1998]; (iv) que os nós utilizem a versão 6 do protocolo de rede IP, conforme especificado no RFC 2460 [Deering 1998]; (v) que os nós implementem o protocolo Mobile IPV6 (MIPv6) como especificado no RFC 3775 [Johnson, Perkins e Arkko 2004]; e (vi) que os nós da rede possam implementar extensões como melhoria ao MIPv6.

O projeto NEWSKY [Schnell e Scalise 2007] foi o pioneiro no desenvolvimento e análise da nova geração de tecnologias baseadas no modelo ATN/IPS. Além das especificações requeridas no documento do ICAO como o uso dos protocolos MIPv6, TCP e Diffserv, o projeto também incluiu a extensão de mobilidade conhecida como NEMO (Network Mobility), o uso de um otimizador de rota (protocolo global HAHA) e o uso do padrão IEEE 802.21 para funcionalidades de handover entre estações aéreo- 
terrestres.

Portanto, de acordo com o manual 9896 e com o projeto NEWSKY, o estabelecimento de uma rede ATN/IPS deve considerar os seguintes protocolos:

- MIPv6 $\rightarrow$ protocolo base da arquitetura ATN/IPS, permitindo mobilidade entre nós móveis e redes IPv6 de acesso. Cada nó móvel deve receber um endereço IP primário (também conhecido como Home of Address ou HoA) e um endereço IP secundário (também conhecido como (Care of Address ou CoA). Uma entidade de rede denominada agente local (Home Agent ou HA) realiza o mapeamento entre endereços primários e secundários de um roteador móvel, redirecionando o fluxo de pacotes para a rede primária e mantendo a comunicação durante a movimentação do nó.

- $\quad$ NEMO $\rightarrow$ Definido no RFC 3963 [Devarapalli et al 2005], esta extensão insere e gerencia uma nova entidade na rede denominada Roteador Móvel (Mobile Router ou MR). Este roteador reduz a sobrecarga de sinalização em enlaces aeronáuticos, mudando e reconfigurando uma rede toda durante o processo de handoff entre estações.

- Diffserv $\rightarrow$ este protocolo descreve, classifica e gerencia o tráfego da rede para suportar a provisão de QoS em redes IP. O protocolo lida com serviços em redes de baixa latência e jitter (e.g. aplicações de voz), em redes sensíveis a atrasos (e.g. redes multimídias) e em redes não sensíveis ao atraso (e.g. documentos).

- Global HAHA $\rightarrow$ O método Global HAHA (Global Home Agent to Home Agent) é um método de otimização de rota para reduzir o atraso fim-a-fim nas redes móveis. Este método distribui HAs ao longo da rota de modo que o MR possa escolher o HA mais próximo, utilizando o protocolo Inter-HAHA para compartilhar informações de mobilidade dos roteadores móveis.

- IEEE 802.21 $\rightarrow$ Este protocolo é responsável por fornecer informações de enlace e de rede para as camadas superiores da arquitetura ATN/IPS, propiciando um melhor gerenciamento dos handovers entre diferentes redes de acesso. O padrão permite handovers entre redes diferentes de acesso ou dentro de uma específica rede de acesso. O protocolo utiliza serviços que permitem que as camadas superiores de uma rede controlem suas camadas inferiores para desempenhar tarefas como reconfiguração e seleção de enlaces apropriados, além de obter informações sobre a conectividade do enlace.

\subsection{Segmentação do TCP}

Em uma rede aeronáutica, o estabelecimento de uma conexão do TCP pode ser feito da seguinte forma: (i) por meio de comunicação direta entre transmissores e receptores; (ii) por intermédio de uma arquitetura DTN; e (iii) por meio de segmentações feitas na rede utilizando a abordagem PEP. Na comunicação utilizando um proxy otimizador de desempenho (Proxy Enhancing Performance ou PEP), um ou mais nós intermediários segmentam a rede em duas (PEP integrado) ou em mais partes (PEP distribuído) [Border 2001]. A abordagem PEP possibilita o uso de um outro protocolo de transporte ou um TCP modificado para suportar trechos na rede com elevadas taxas de ruído ou com baixa qualidade do sinal. A comunicação baseada em PEP aumenta o desempenho do TCP, mas dificulta o uso de protocolos relacionados a segurança da rede. Tais segmentações devem ser implementadas para aumentar o desempenho dos serviços de comunicação para passageiros aéreos. 


\section{Abordagem SynFlight}

A abordagem SynFlight [Silva et al. 2014] consiste em construir um sistema de comunicação para reduzir as perdas de desempenho na comunicação aéreo-terrestre em rotas aéreas sem uma cobertura de rede adequada. A transmissão pode ser controlada através de temporizadores ou por eventos específicos que ocorram na rede, programando os períodos que haverão comunicação entre a aeronave e as estações aéreo-terrestres e os que não haverão. Para tal programação, o sistema estabelece entidades de rede que inferem informações de um plano de voo tais como velocidade programada de cruzeiro, cobertura de uma estação ATG e posicionamento atual da aeronave em relação às estações. O sistema SynFlight apresenta as seguintes características:

- Sistema projetado para tolerar atrasos e interrupções na comunicação ao longo de uma rota aérea;

- A arquitetura do sistema utiliza a suíte moderna de protocolos para redes aeronáuticas (PMIPv6 / NEMO) e é centrada na rede, sendo os serviços oferecidos de forma transparente aos usuários finais (i.e. o equipamento dos usuários não é alterado);

- Adota-se uma segmentação entre a rede aviônica e a rede terrestre através do método PEP distribuído;

- O SynFlight gerencia um conjunto específico de entidades de rede que processam as informações obtidas em um plano de voo e programam as transmissões no canal aeronáutico;

- O sistema permite o uso de diversos métodos para a otimização de desempenho do TCP, sendo os principais citados a seguir: (i) ajustes adaptativos ou temporários de parâmetro (técnica denominada SFL1); e (ii) congelamento de janelas de transmissão (técnica denominada SFL2).

Basicamente, o Synflight introduz uma sincronização entre a posição da aeronave e a condição de transmissão, mantendo a sessão do TCP estável durante a rota e aumentando, por consequência, a vazão desta transmissão. Para que a sincronização ocorra, inserem-se 2 agentes de rede no roteador móvel do sistema aviônico e dois protocolos neste roteador e no HA da porção terrestre da rede. Leva-se em conta o ambiente aeronáutico em que o sistema está inserido além de informações da camada de enlace do roteador móvel para saber quando um canal de comunicação está disponível para transmissões.

\subsection{Arquitetura}

A arquitetura do sistema SynFlight é baseada no padrão ATN/IPS, utilizando os seguintes protocolos [Schnell and Scalise, 2007, Bauer and Ayaz 2008]:

- Arquitetura Proxy Mobile IPv6 (PMIPv6) ;

- Network Mobility (NEMO);

- Protocolo Diffserv para QoS;

- IEEE 802.21 para enlaces aéreo-terrestres;

- Tecnologia 4G (Long Term Evolution ou LTE); 
- Metodologia Global HAHA para otimização de rotas;

A tecnologia LTE [Dusza et al. 2013] é adotada como sendo o padrão de comunicação entre a aeronave e as estações devido a sua elevada largura de banda e boa capacidade de transmissão com veículos de alta velocidade. Note que a rede aeronáutica é segmentada em 3 sub-redes através da utilização de um PEP distribuído entre o HA e o roteador móvel. Tal segmentação permite que as modificações necessárias para o funcionamento do sistema seja a nível de rede, proporcionando transparência aos usuários finais. A Figura 1 ilustra a pilha de protocolos utilizadas pela arquitetura SynFlight.

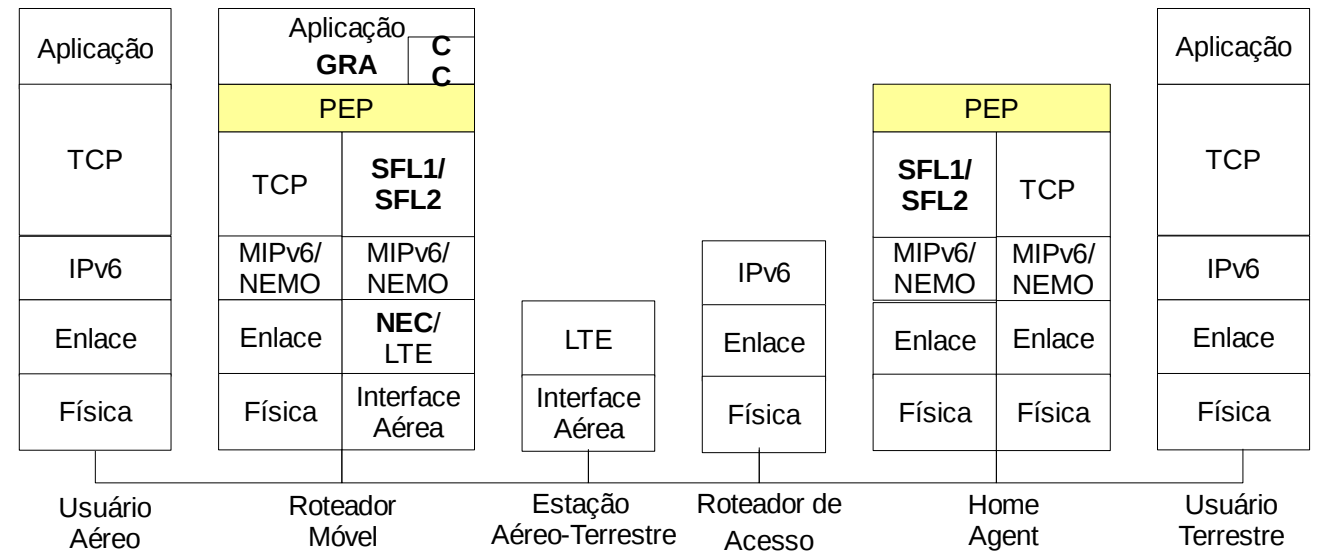

Figura 1. Pilha de Protocolos da Abordagem SynFlight

\subsection{Entidades da Rede}

O sistema SynFlight é composto por quatro entidades de rede que permitem o sincronismo entre a posição da aeronave e o inicio de uma desconexão de enlace, ativando no instante correto os parâmetros do TCP. Tais entidades são formadas por um agente notificador do estado de conectividade (NEC), um agente gerenciador da rota aérea (GRA), um protocolo TCP modificado (CET) e um protocolo de convergência (CC) [Silva et al. 2014]. A Figura 2 ilustra a inserção destas entidades na topologia de rede aeronáutica.

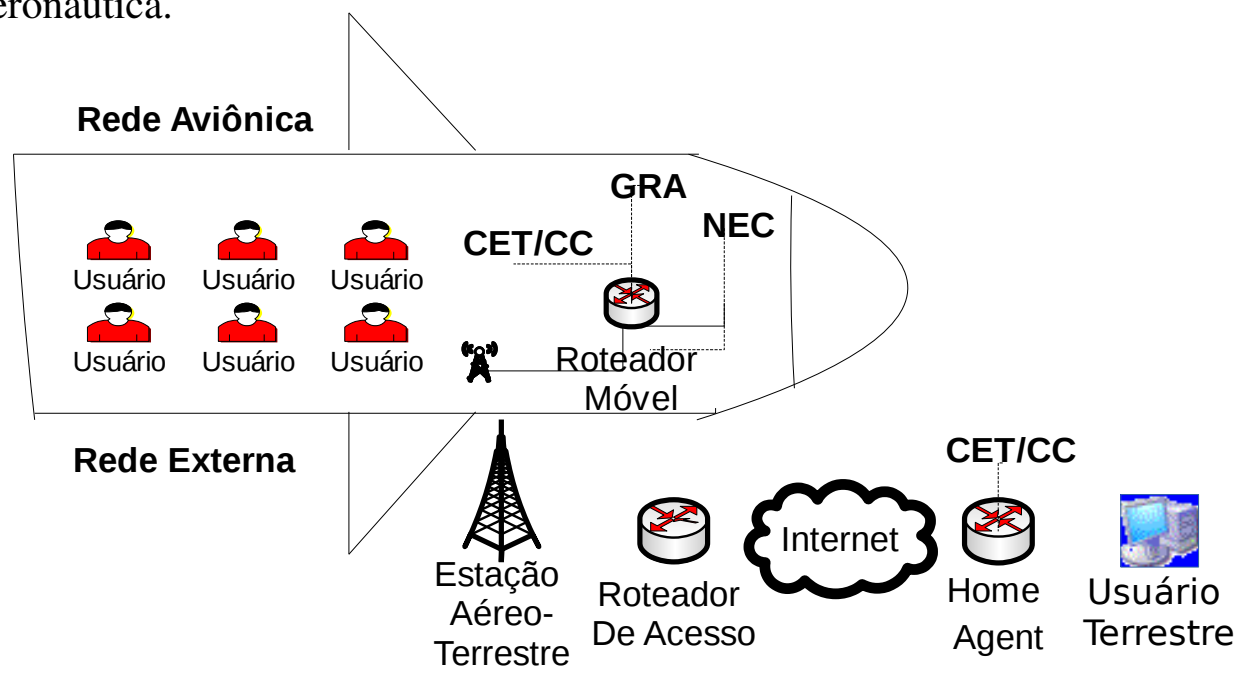

Figura 2. Topologia da Rede com Entidades SynFlight Associadas 


\subsubsection{Entidade NEC}

A entidade NEC situa-se no sistema aviônico e é responsável por distribuir informações sobre o estado de conectividade entre estações e aeronaves para outras entidades do sistema. As informações consistem em mensagens referentes ao estado atual do enlace (i.e. se o enlace encontra-se disponível para transmissão ou não) e mensagens contendo o endereço MAC da atual estação, utilizados pela entidade GRA para estimar o posicionamento atual da aeronave (i.e. em que estação ATG a aeronave está sobrevoando). A entidade CET utiliza tais informações para realizar a mudança de estado em sua máquina de estados. O protocolo IEEE 802.21 [Schnell and Scalise 2007] é utilizado como base para prover os serviços ofertados por esta entidade.

\subsubsection{Entidade GRA}

A entidade GRA é responsável por gerenciar informações da rota aérea definidas no plano de voo, predizendo os períodos de transmissão e de interrupção e processando mensagens de/para outras entidades do sistema. Tal entidade é dividida em quatro módulos principais:

- Plano de voo: contém informação relevante sobre a rota programada (aerovias escolhidas) e sua situação atual (i.e. a posição momentânea da aeronave, sua distância percorrida na rota e quantas estações ou interrupções faltam ser percorridas), o aeródromo de origem e o de destino, a velocidade de cruzeiro pretendida e a duração do voo.

- Estimador de temporizadores: estima os períodos de transmissão e interrupção que ocorrerão em uma rota aérea, processando informações obtidas na base de informações aeronáuticas e no plano de voo.

- Gerenciador de mensagens: é responsável por receber e processar mensagens vindas da entidade NEC e por preparar e enviar mensagens contendo a estimativa dos temporizadores para a máquina de estados implementada no protocolo TCP modificado (entidade CET).

- Base de informações aeronáuticas: contém um mapa da distribuição das estações ATG ao longo da rota aérea com as posições geográficas das estações e seus respectivos endereços físicos, além de informações do estado destas estações (e.g. tamanho de cobertura da rede ou da potência do sinal, estações defeituosas, estações com restrições de energia ou desativadas, posicionamento das antenas nas estações). Esta base também pode ser preenchida com laudos sobre alguma condição específica no ambiente (e.g. laudos metereológicos que podem ajudar no ajuste da velocidade de cruzeiro em uma aeronave).

Depois que programar a rota aérea a partir da estimação dos temporizadores de transmissão e interrupção, cria-se uma tabela de rota programada com os valores estimados. A entidade GRA deve controlar esta tabela e utilizar o gerenciador de mensagens para enviar tais informações a entidade CET, o qual instancia ambos temporizadores durante o percorrer da rota.

\subsubsection{Entidade CET}

A entidade CET é responsável por estabelecer o sincronismo da transmissão entre os 
TCPs modificados no roteador móvel e nos agentes locais, aumentando a capacidade de transmissão da rede. Tal entidade é dividida em três módulos principais:

- Máquina de Estados: controla a transmissão, permitindo reduzir as retransmissões e os atrasos de reinicio na comunicação. Esta entidade instancia e controla os temporizadores de transmissão e interrupção ao longo da rota aérea.

- Ajustador de Parâmetros TCP: este módulo efetua alterações nos parâmetros dos TCPs modificados para otimizar o desempenho da rede aeronáutica.

- Gerenciador de Mensagens: este módulo recebe, envia e processa mensagens de sinalização entre os TCPs modificados. O módulo recebe mensagens da entidade NEC sobre o estado de conectividade do enlace, necessário para se realizar a mudança entre os estados implementados na máquina de estados.

A máquina de estados da entidade CET elabora 3 estados para controlar o TCP em cenários aeronáuticos: (i) estado de transmissão; (ii) estado de interrupção não programada; e (iii) estado de interrupção programada. A máquina de estados é adaptada ao estado de transmissão do TCP, conforme ilustrado na Figura 3.

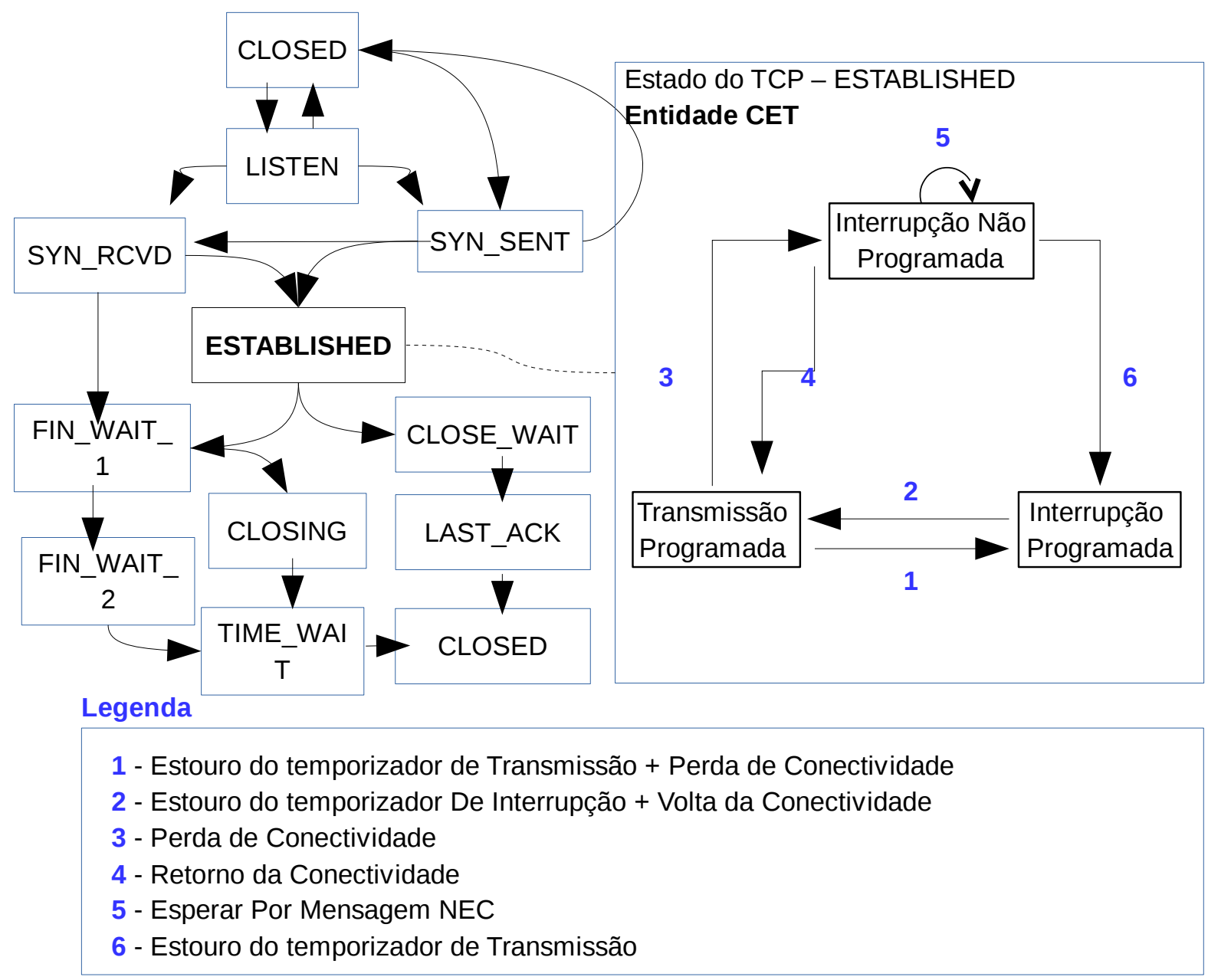

Figura 3. Adaptação na Máquina de Estados do TCP tradicional

O estado de transmissão programada permite que os dados sejam transmitidos durante o sobrevoo de uma aeronave em uma estação ATG. Neste estado, é possível 
atualizações sobre a rota e envio de mensagens de sinalização para sincronizar o sistema aviônico com o sistema terrestre. Um temporizador é estabelecido para definir o tempo de vida deste estado. Já o estado de interrupção não programada é ativado quando o enlace perde sua conectividade durante a fase de transmissão. Neste caso, não há transmissão e o estado só deverá mudar quando o enlace detectar novamente a conectividade. Por último, o estado de interrupção programada é ativado quanto não há conectividade no fim da fase de transmissão. Durante esta fase, o temporizador de interrupção é ativado e os ajustes nos parâmetros do TCP podem ser realizados.

As mudanças de parâmetros no TCP são feitas de duas formas: (i) ajustando o parâmetro do temporizador de retransmissão do TCP (denominado SynFlight1 ou SFL1); (ii) ajustando o parâmetro da janela de transmissão do TCP (denominado SynFlight2 ou SFL2). O mecanismo SFL1 adapta o temporizador de retransmissão (RTO) durante a fase de interrupção. Quando uma aeronave está saindo da cobertura de rede de uma estação e indo em direção a uma próxima estação, o valor do RTO é configurado para ser o tempo previsto que uma aeronave percorrerá a interrupção.

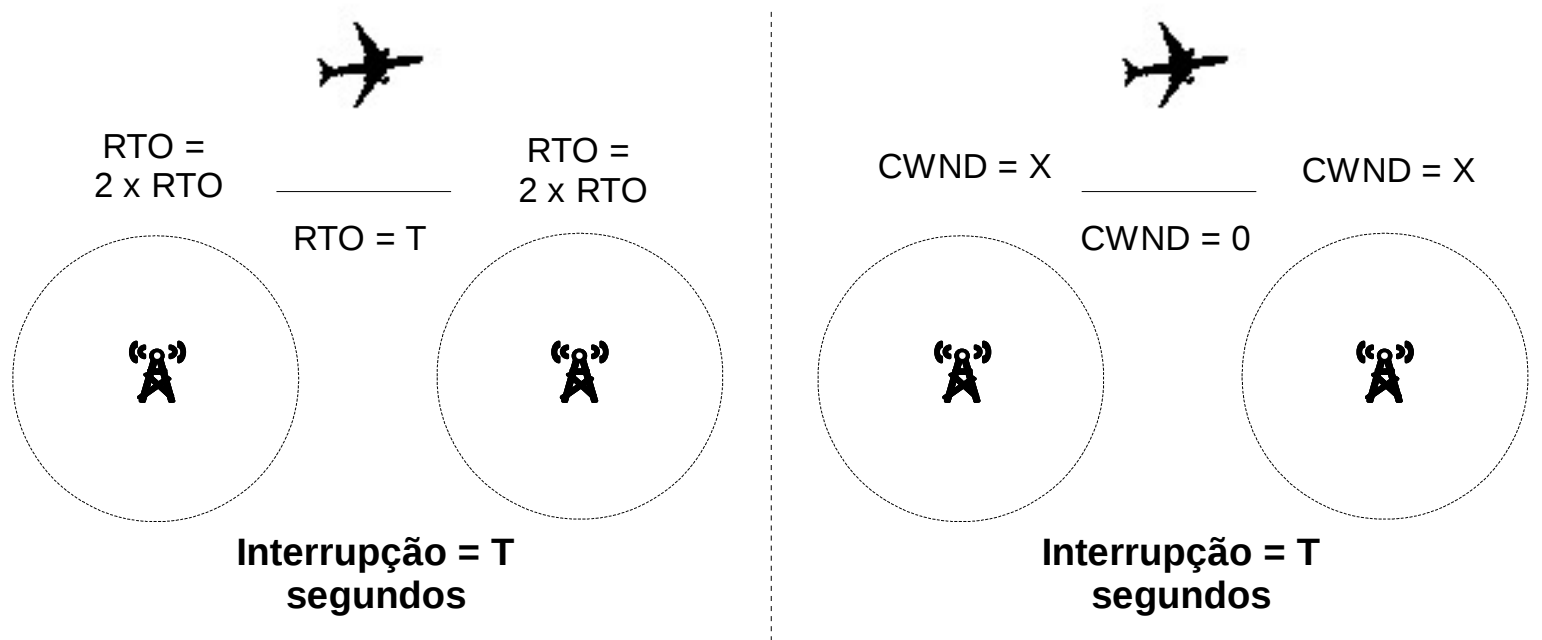

Figura 4. Técnicas SFL1 (esquerda) e SFL2 (direita)

Portanto, o RTO será igual ao temporizador de interrupção calculado pela entidade GRA. Caso o RTO expire e seja necessária uma nova retransmissão, a técnica deve retornar o valor anterior do RTO, pois, caso contrário, tal adaptação implicará em altos valores de atrasos de reinício. O mecanismo SFL2 congela os parâmetros de retransmissão do TCP, mudando a janela de congestionamento para zero durante o período de interrupção. Deste modo, não há retransmissões desnecessárias e o último valor da janela de transmissão pode permanecer inalterado.

\subsubsection{Entidade CC}

Esta entidade funciona como uma camada intermediária para estabelecer a comunicação entre as entidades GRA e CET. A principal função desta entidade é garantir o repasse das notificações elaboradas por outras entidades, como as mensagens com os valores dos temporizadores e as mensagens que contenham informações sobre o estado de conectividade do enlace. O desenvolvimento desta entidade é simples, sendo instalada em um roteador móvel e colocada acima da camada de transporte (overlay). 


\subsection{Mensagens de Sinalização}

As entidades propostas neste trabalho comunicam-se por meio de mensagens ICMP, empregando o método cross layer entre as entidades. A entidade NEC fica monitorando o canal continuamente ao longo do percorrer de uma rota aérea. Ao perceber a mudança de status da conectividade, a entidade envia a mensagem de status para a entidade GRA.

A entidade GRA processa os valores dos temporizadores de transmissão e da próxima interrupção, repassando a mensagem de status para a entidade CET primária, responsável em guardar tais valores em uma variável e utilizá-las quando for conveniente. Neste caso, o temporizador de transmissão da máquina CET é ajustado e o temporizador da próxima interrupção é armazenado para quando houver tal interrupção. Isto permite que a técnica SFL1, por exemplo, substitua o RTO do seu mecanismo de retransmissão por este valor armazenado. Por último, o CET primário envia tal pacote para que o CET secundário também armazene este valor em uma agenda para uso posterior. Inicia-se, portanto, a transmissão sincronizada do TCP até que o temporizador da transmissão seja expirado. Caso hajam mudanças de rota ou nas informações definidas na entidade GRA, a entidade CET é responsável em realizar tais atualizações em sua entidade secundária, durante o processo de transmissão.

A mudança de estado da transmissão do TCP só ocorrerá quando a entidade NEC enviar o pacote contendo a informação sobre o enlace desconectado e o temporizador de transmissão for expirado. Nesta fase de interrupção programada, não há troca de informações entre os CETs, apenas a aplicação da técnica SFL1 ou SFL2 para reduzir os efeitos negativos gerados pelo mecanismo de retransmissão no TCP. A transmissão só retorna quando a entidade NEC enviar um novo pacote contendo a informação sobre o enlace conectado.

$\mathrm{Na}$ fase de interrupção não programada. o temporizador de transmissão ainda está ativo e uma informação de não conectividade é enviada pela entidade NEC. A partir do momento em que a entidade NEC envia tal informação, o TCP deve zerar sua janela de congestionamento e ficar no aguardo do retorno de conectividade. Caso a entidade NEC não envie e o temporizador de transmissão expirar, a máquina de estados CET muda seu estado para interrupção programada, assumindo valores definidos a priori. Este estado permite que o TCP obtenha desempenhos superiores quando as interrupções não forem previstas durante a fase de transmissão. Isto permite que não haja retransmissão e que a janela de congestionamento permaneça inalterada após o retorno da transmissão. Note que este estado só pode ser utilizado pela técnica SFL2.

\section{Análise de Desempenho}

Este artigo elabora uma simulação baseada em eventos discretos para analisar o comportamento dos protocolos TCP, DTN e da abordagem Synflight na presença de interrupções aeronáuticas. Os mecanismos simulados são implementados em um simulador específico para redes aeronáuticas denominado ANS. Este simulador foi projetado por pesquisadores da Universidade de Salzburg para avaliar partes do projeto SANDRA [Ehammer e Rokitansky 2011]. O ANS é baseado na pilha de protocolos MIPv6, permitindo o desenvolvimento da topologia de rede aeronáutica, o modelo de dados e mobilidade, funções da rede e seus mapeamentos, entidades e avaliação de desempenho. A metodologia da simulação é descrita a seguir.

\subsection{Metodologia da Simulação}


O processo de simulação dos protocolos é definido em duas etapas. Na primeira etapa, utiliza-se o simulador ANS para gerar dados sobre o comportamento dos mecanismos de transmissão do TCP, DTN e do SynFlight ao longo do cenário investigado. Utiliza-se as camadas 2,3 e 5 do simulador, sendo implementada a camada 4 (TCP, DTN e SynFlight) para prover a transmissão aeronáutica. Os resultados desta simulação são armazenados em arquivos de saída. Na segunda etapa, utiliza-se a ferramenta MATLAB para processar estes arquivos de saída, organizá-los e imprimi-los em forma de gráficos.

\subsection{Configuração}

A configuração do cenário aeronáutico é descrito como segue. Para fins de simulação, utiliza-se duas estações ATG com cobertura de rede simétrica e com uma distância típica entre estações alocadas em cidades vizinhas. Considera-se que a perda de enlace ocorrerá exatamente no momento em que a aeronave alcançar o limite de cobertura da primeira estação. A partir deste ponto, o temporizador de retransmissão do TCP será expirado várias vezes até alcançar a cobertura de rede da nova estação ATG e seu temporizador expirar, voltando a transmitir os pacotes. A Figura 5 ilustra o comportamento desta simulação. As principais características de configuração e parâmetros são descritos a seguir:

- A simulação realizada neste trabalho utiliza a técnica SFL1 do SynFlight;

- Adota-se o TCP NewReno [Floyd, Gurtov e Henderson 2004] para fins de simulação, com parâmetro RTT fixo;

- A distância simulada entre as estações ATG é de $83 \mathrm{~km}$, representando a distância física real entre as cidades de Congonhas e Belo Horizonte, ambas localizadas no estado de Minas Gerais;

- A cobertura de rede mede 50 km em cada estação ATG;

- O parâmetro T representa o tempo de interrupção estimado pela entidade GRA;

- O parâmetro MaxRTO representa o limiar para realizar a mudança do comportamento exponencial para o linear do mecanismo de retransmissão do TCP;

- O parâmetro WNDtrans representa a janela de transmissão responsável pelo controle de congestionamento do TCP. Este parâmetro atua nos estados de slow start (SS), congestion avoidance (CA) e fast recovery (FR).

- O parâmetro WNDrec representa a janela de recepção responsável pelo controle de fluxo do TCP. Este parâmetro também atua nos estados SS, CA e FR.

- O parâmetro WNDmax representa a janela máxima de transmissão permitida pelo sistema, sendo definida pelo produto atraso-largura de banda da rede.

- O parâmetro Ssthresh corresponde ao limiar necessário para a mudança de comportamento do controle de congestionamento do TCP;

- Utiliza-se o enlace LTE para fins de simulação. O canal investigado é do tipo uplink, sendo as antenas das estações direcionadas para o céu (skyward).

- O modelo de energia do enlace LTE descrito em [Dusza et al 2013] serviu como referência para calcular o consumo de energia de um roteador móvel aeronáutico. O consumo é calculado em Watts durante o percorrer da aeronave. 

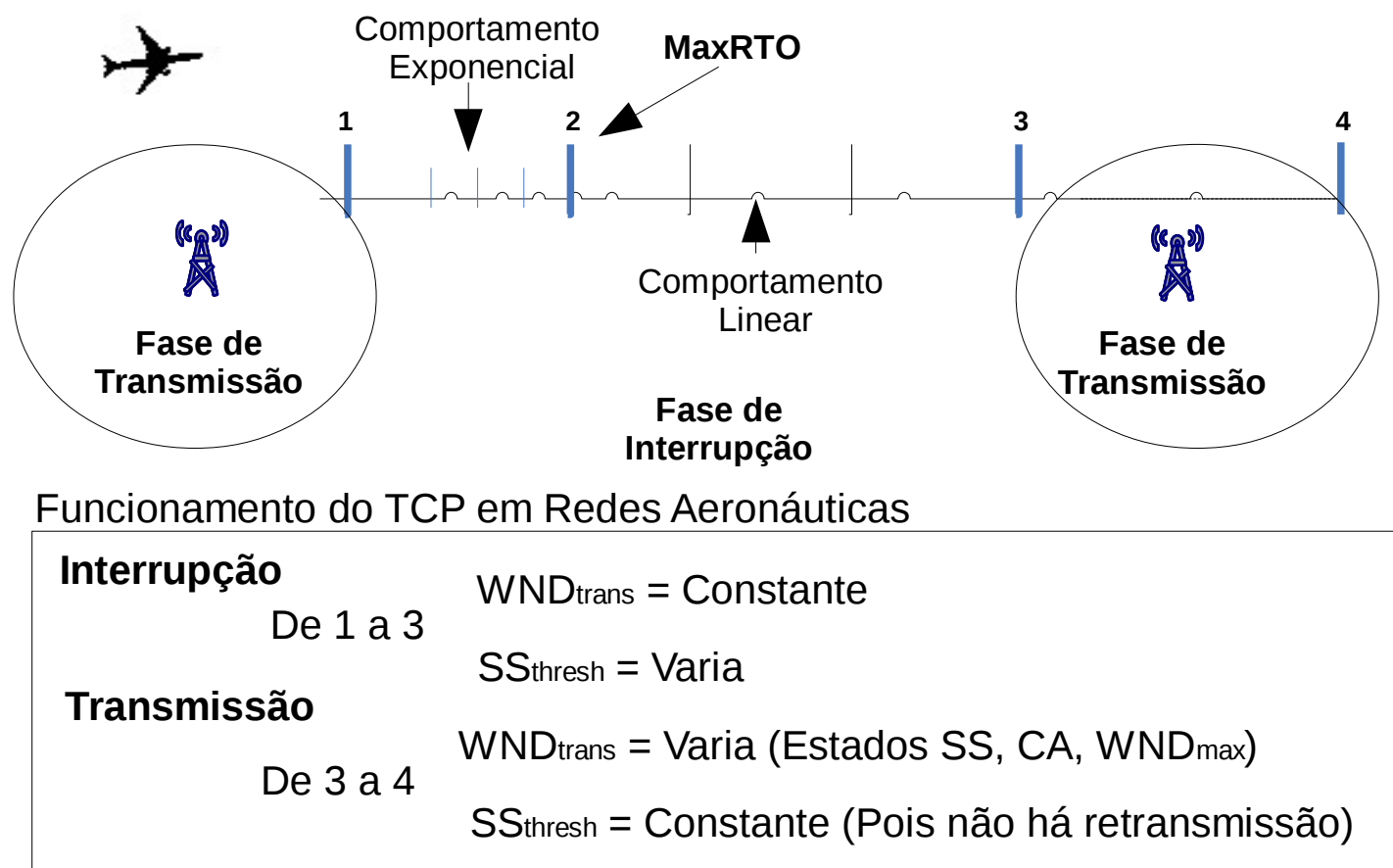

Figura 5. Cenário da Simulação

Note que, durante o período de interrupção, as retransmissões provocadas pelos mecanismos TCP e SFL1 variam o parâmetro Ssthresh e a janela de transmissão do TCP permanece constante (depois de ser reduzida para 1 segmento quando houver a primeira retransmissão).

\subsection{Resultados}

Esta seção apresenta os resultados obtidos pela simulação dos protocolos TCP, DTN e SynFlight, realizadas no simulador ANS.

A Figura 6(a) representa o número médio de retransmissões obtidos na simulação. $\mathrm{O}$ eixo $\mathrm{Y}$ representa a quantidade de retransmissões realizadas em um trecho aeronáutico com interrupções. Note que as maiores retransmissões são obtidas pelo protocolo DTN, devido a sua peculiar característica de encerrar rapidamente a sessão TCP e tentar estabelecer uma nova conexão através de pacotes SYN. O algoritmo DTN aumenta o número de retransmissões em detrimento a uma maior tolerância na comunicação. O SynFlight obtém o melhor desempenho em relação ao TCP e ao DTN devido ao sincronismo realizado por ele através de um plano de voo, garantindo uma minimização destas retransmissões.

A Figura 6(b) representa o gasto de energia realizado pelos protocolos simulados. O eixo Y representa o consumo médio de energia (em Watts). Note que os protocolos TCP e DTN gastam mais energia do que o mecanismo SynFlight. Isto ocorre porque o gasto de energia é proporcional ao número de retransmissões.

A Figura 6(c) representa o cálculo da capacidade de transmissão obtido pela simulação. As mensagens de sinalização não são consideradas nesta investigação, sendo avaliado apenas o goodput médio (em MB/s). A vazão inicial de uma estação ATG depende do 


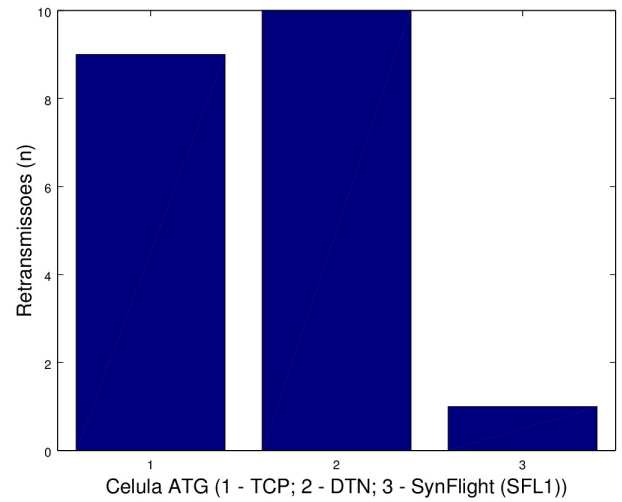

(a)

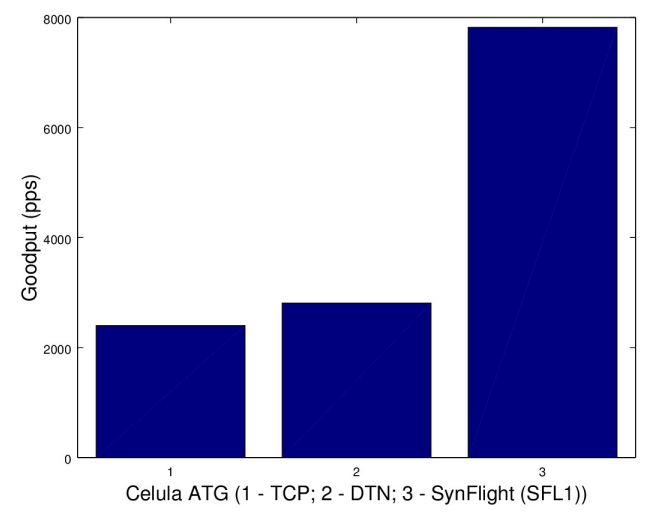

(c)

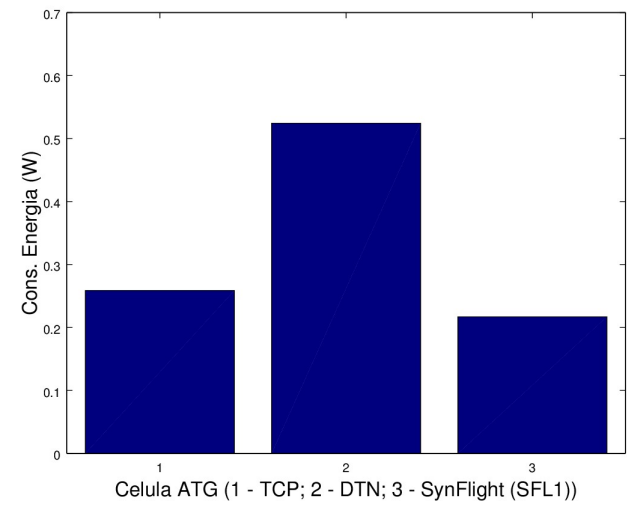

(b)

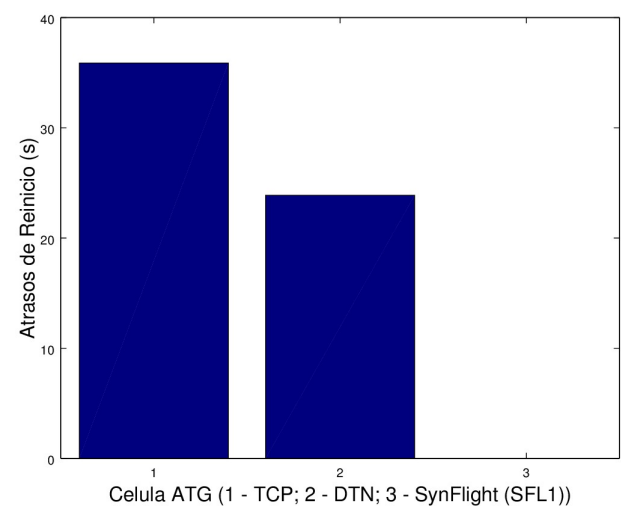

(d)

Figura 6. Resultados da Simulação

desempenho do mecanismo de retransmissão na estação anterior. Quanto menor for o número de retransmissões, maior será o patamar do slow start, permitindo uma maior recuperação no reinício da comunicação. Por outro lado, a abordagem SynFlight insere um ou nenhum pacote durante a fase de interrupção, impedindo que o patamar do slow start seja fortemente modificado e evitando o atraso de reinício, obtendo um desempenho maior de transmissão do que os protocolos TCP e DTN.

A Figura 6(d) representa o atraso de reinício obtidos pelos protocolos na simulação. O eixo Y representa o atraso médio de reinício (em segundos). O mecanismo SynFlight obtém atrasos insignificantes, pois o mesmo se beneficia do sincronismo entre transmissores e receptores para rapidamente reagir e retransmitir os dados em tempo hábil, aumentando a capacidade de transmissão da rede. Por outro lado, os protocolos TCP e DTN geram atrasos que retardam o início da comunicação, prejudicando a transmissão em célula.

\subsection{Discussão}

A utilização do SynFlight em redes aeronáuticas aumenta o desempenho destas redes em cenários com interrupções frequentes. A escolha das técnicas SFL1 ou SFL2 dependerá do tipo de ambiente encontrado. Na técnica SFL1, a redução dos atrasos de reinício e das retransmissões está ligado com a eficiência do sincronismo entre transmissores e receptores em um trecho aeronáutico interrompido. $\mathrm{O}$ sincronismo deve 
ocorrer quando não houver variações significativas na velocidade da aeronave. Quanto menor for o erro de sincronismo, maior será a redução nos atrasos e nas retransmissões. A técnica SFL1 é simples, de fácil processamento e não necessita de uma entidade NEC instalada. Na técnica SFL2, não há necessidade de sincronismo, pois a janela de congestionamento, em um trecho interrompido, estará congelada até quando a máquina de estados receber uma notificação do estado de enlace, decidindo pela mudança de estado. A eficiência, neste caso, estará ligada ao agente NEC, pois este decidirá quando o parâmetro CWNDtrans será alterado. Esta técnica não é afetada pelos erros de sincronismo da máquina de estados, porém exige um maior processamento devido a necessidade de se controlar pacotes de keep alive.

A entidade CET é responsável em proporcionar a tolerância a interrupções, não sendo necessária a implementação de uma camada DTN na arquitetura de rede aeronáutica. Esta entidade se beneficia do método PEP para fazer a sincronização entre os segmentos da rede, ao invés do protocolo BP. Não há necessidade de store and forward, o que não causa sobrecarga do roteador móvel nem do home agent. Portanto, o protocolo DTN é utilizado neste trabalho apenas para fins de comparação. As entidades NEC e GRA servem de auxílio para que a entidade CET cumpra a sua tolerância eficazmente.

\section{Conclusão}

Este artigo investigou o desempenho dos protocolos TCP, DTN e a abordagem SynFlight no cenário típico aéreo-terrestre. Os resultados indicaram que a abordagem SynFlight obteve ganhos significativos de desempenho em relação aos mecanismos convencionais de retransmissão. O SynFlight reduziu o impacto do mecanismo de retransmissão do TCP, garantindo uma redução nos atrasos de reinício, nas retransmissões, propiciando um melhor consumo de energia e uma vazão superior. Os protocolos DTN e TCP não têm esse poder de desempenho, sofrendo com os atrasos de reinício e retransmissões, reduzindo suas janelas de transmissão e aumentando a espera para iniciar a transmissão dos dados.

\subsection{Propostas para padronização pela IETF}

As propostas para padronização pela IETF consistem nos seguintes pontos:

1. Padronização da Arquitetura $\rightarrow$ A IETF tem documentos do tipo informativo que servem para formalizar arquiteturas, plataformas, entre outros. Neste caso, a arquitetura SynFlight poderia ser padronizada utilizando suas entidades de rede e a integração entre os vários protocolos IETF envolvidos.

2. Padronizar a entidade CET $\rightarrow$ Esta entidade requer regras para atualização de estados da máquina TCP e para solicitação e recebimento de mensagens vindas de outras entidades.

3. Padronizar a entidade $\mathrm{CC} \rightarrow$ Esta entidade requer regras para realizar a comunicação entre a entidade CET e uma aplicação controladora (e.g. GRA).

4. Padronizar a entidade GRA $\rightarrow$ Esta entidade requer um alto nível de estimação de temporizadores e análise de informações sobre a rota, devendo suas entradas, seu processamento e suas saídas também serem padronizadas. 


\section{Referências}

Silva, R.A., Netto, J.E., Paiva, M.A.A. e Anzaloni, A. (2014) "SynFlight: Uma abordagem de Transmissões Programadas Atenta a Interrupções para Redes AéreoTerrestres", In IEEE Latin America Transactions, Dezembro, IEEE publisher.

Ott, J., Demmer, M. e Perreault, S. (2014) "Delay-Tolerant Networking TCP Convergence-Layer Protocol ", RFC 7242, IETF Standard.

Jacobson, V., Braden, B., e Borman, D. (2014) “TCP extensions for high performance ", RFC 7323, IETF Standard.

Dusza, B., Ide, C., Bok, P.B. e Wietfeld, C. (2013) "Optimized Cross-layer protocol choices for LTE in High speed vehicular environments", In Wireless Communications and Mobile Computing Conference, IEEE Publisher, Piscataway.

Ehammer, M. e Rokitansky, C.H. (2011) "Running IPv6 over Aeronautical Links", tese de doutorado, Universität Salzburg.

ICAO (2011) "Manual for the ATN using IPS Standards and Protocols", Doc 9896, ICAO draft.

Caini, C., Cruickshank, H., Farrell, S., e Marchese, Mario. (2011) "Delay and Disruption-tolerant networking (DTN): an alternative solution for future satellite networking applications". In Procedings of the IEEE, IEEE publisher, Piscataway.

Caini, C., Cornice, P., Firrincieli, R., Livini, M. e Lacamera, D. (2009) “Analysis of TCP and DTN retransmission algorithms in presence of Channel Disruptions", In International Conference on Advances in Satellite and Space Communications, IEEE Publisher, Piscataway.

Bauer, C. e Ayaz, S. (2008) "A thorough investigation of Mobile IPv6 for the Aeronautical environment", In Vehicular Technology Conference, IEEE, Piscataway.

Schnell, M. and Scalise, S. (2007) "NEWSKY: Concept for Networking the SKY for Civil Aeronautical Communications", In Aerospace and Electronic Systems Magazine, IEEE publisher, Piscataway.

Devarapalli, V., Wakikawa, R., Petrescu, A. e Thubert, P. (2005) "Network Mobility (NEMO) Basic Support Protocol", RFC 3963, IETF Standard.

Floyd, S., Gurtov, A. e Henderson, T. (2004) “The NewReno modification to TCP's fast recovery algorithm ", RFC 2582, IETF Standard.

Johnson, D., Perkins, C. e Arkko, J. (2004) "Mobility support in IPv6", RFC 3775, IETF Standard.

Fall, K. (2003) “A delay-tolerant network architecture for challenged internets", In Conference on applications, technologies, architectures, and protocols for Computer Communications, ACM publisher, New York.

Border, J (2001) "Performance Enhancing Proxies Intended to Mitigate Link Related Degradations", RFC 3135, IETF Standard.

Blake, S., Black, D., Carlson, M., Davies, E., Wang, Z e Weiss, W (1998), “An Architecture for Differentiated Services ”, RFC 2475, IETF Standard.

Deering, S. (1998) "Internet protocol, version 6 (IPv6) specification", RFC 2460, IETF. 